\title{
PREVALÊNCIA DE DISCINESIA TARDIA EM PACIENTES INTERNADOS NOS HOSPITAIS PSIQUIÁTRICOS DE SALVADOR, BAHIA, BRASIL
}

\author{
A.S. ANDRADE FILHO*, S.R.S. SANTOS**, C.L. MEDEIROS**, C.C. CARNEIRO**, \\ F.L.S. FIGUEIROA ${ }^{\star \star}$, M.C. SILVA ${ }^{\star \star \star}$, W.A. DUNNINGHAM ${ }^{\star \star \star \star}$
}

\begin{abstract}
RESUMO - Os autores apresentam estudo transversal da prevalência da discinesia tardia (DT) em pacientes internados em unidades psiquiátricas da cidade de Salvador durante o mês de setembro-1992. Após revisão da literatura, comentam a prevalência média de DT encontrada: $1,65 \%$ na população de 2115 pacientes, usando protocolo baseado na escala de Simpson.
\end{abstract}

PALAVRAS-CHAVE: discinesia tardia, prevalência, neurolépticos.

\section{The occurrence of tardive dyskinesia on inpatients from Salvador, Bahia, Brazil}

SUMMARY - The authors present a cross-sudy on the occurrence of tardive dyskinesia (TD) among inpatients of psychiatric units of the city of Salvador during the month of September, 1992. After reviewing the literature, they comment the average prevalence found for TD: $1,65 \%$ in a population of 2,115 patients using a protocol based on the Simpson Scale.

KEY WORDS: tardive dyskinesia, prevalence, neuroleptic drugs.

O aparecimento de clorpromazina, na década de 50, trouxe enorme avanço e esperança no tratamento dos distúrbios psiquiátricos. Desde então, o número de substâncias neurolépticas sintetizadas aumentou consideravelmente, trazendo benefícios no tratamento das psicoses, principalmente reduzindo o período de internação dos pacientes ${ }^{3}$. No entanto, logo após a introdução das drogas neurolépticas, sinais extrapiramidais foram observados precocemente no tratamento, desaparecendo com a descontinuação da droga, sendo aliviados por medicamentos antiparkinsonianos $^{6}$. Na própria década de 50, movimentos discinéticos involuntários, às vezes persistentes, foram encontrados tardiamente no tratamento com os neuroléticos ${ }^{6}$. Foram inicialmente denominados de "síndrome bucolingomastigatória" e, subsequentemente, de "síndrome de insuficiência extrapiramidal terminal". O termo discinesia tardia (DT) (discinesia de início tardio e persistente), correntemente utilizado, foi aplicado pela primeira vez em 1964 por Faurbye e col., segundo Marsden?. Trata-se de síndrome vinculada ao uso prolongado de neurolépticos, que se caracteriza pelo aparecimento de movimentos hipercinéticos involuntários, afetando predominantemente a língua, lábios e outros músculos faciais, frequentemente acompanhados por movimentos coreoatetósicos, com envolvimento de pernas e tronco ${ }^{2,8,10,11}$. Presume-se que seja causada

Hospital Universitário Professor Edgard Santos (HUPES), Universidade Federal da Bahia (UFBA): *Professor Adjunto, Chefe do Serviço de Neurologia da HUPES-UFBA; **Médicos Residentes em Psiquiatria e em Neurologia no HUPES-UFBA; ***Estagiária do Serviço de Psiquiatria do HUPES-UFBA; ${ }^{* * * *}$ Chefe do Serviço de Psiquiatria do HUPES-UFBA. Estudo apresentado ao 9th World Congress of Psychiatry, June 6-12, 1993, Rio de Janeiro, Brasil. Aceite: 4-janeiro-1994.

Dr. Antonio S. Andrade Filho - Rua Ayrosa Galvão 89 - 40140-180 Salvador BA - Brasil. 
por hipersensibilidade dopaminérgica no sistema nigrostriatal, induzindo por neurolépticos' ${ }^{\prime}$ A DT é a mais importante complicação do uso prolongado de neurolépticos. O que inicialmente se pensava ser curiosidade clínica, tem-se tornado problema de saúde pública significante, devido às implicações clínicas, psicossociais e médico-legais de sua ocorrência ${ }^{8}$. A taxa de incidência na literatura varia entre $0,5 \%$ e $65 \%$ (com a média de 10 a $20 \%$ ) dos pacientes em uso crônico de antipsicóticos ${ }^{10}$, observando-se nos últimos anos aumento progressivo de sua prevalência nos pacientes internados 5 .

Neste estudo, é avaliada a ocorrência de discinesia tardia em pacientes internados nos hospitais psiquiátricos da Cidade de Salvador, Bahia, analisando idade e sexo dos pacientes.

\section{CASUÍSTICA E MÉTODO}

Seis hospitais psiquiátricos e um hospital geral dotado de enfermaria psiquiátrica foram visitados pelos autores durante o mês de setembro-1992. Os pacientes com quadro clínico sugestivo de DT foram previamente selecionados pelos autores, que excluíram os casos duvidosos e incompatíveis. Aqueles pacientes com DT foram submetidos a exame pela Escala de Simpson ${ }^{7}$ (Anexo 1), aplicada por um único observador para evitar variações e interpretações subjetivas. O critério estatístico utilizado para confirmar DT foi pontuação acima de $3 \mathrm{em}$, pelo menos, um dos segmentos.

Anexo I.Questionário de avaliação de movimentos involuntários anormais (QMIA). Variável Neurológica: Escala abreviada para avaliação de discinesia, Rockland Research Institute (Simpson).

Paciente

Data

Local

Pesquisador

Movimentos orofaciais:

1. Área periocular

2. Movimentos dos lábios

3. Movimentos mastigatórios

4. Sinal do bombom

5. Protusão da língua

6. Tremor e/ou movimentos coreoatetósicos da língua

Pontos

Pescoço e tronco:

7. Hipercinesia axial

8. Movimentos rotatórios

9. Movimentos de contorção

\begin{tabular}{llllll}
\multicolumn{7}{c}{ Pontos } \\
1 & 2 & 3 & 4 & 5 & 6 \\
1 & 2 & 3 & 4 & 5 & 6 \\
1 & 2 & 3 & 4 & 5 & 6 \\
1 & 2 & 3 & 4 & 5 & 6 \\
1 & 2 & 3 & 4 & 5 & 6 \\
1 & 2 & 3 & 4 & 5 & 6
\end{tabular}

Extremidades:

10. Movimentos coreatetósicos dos dedos e punhos

11. Movimentos coreatetósicos dos artelho e tornozelos

12. Bater os pés

$\begin{array}{llllll}1 & 2 & 3 & 4 & 5 & 6 \\ 1 & 2 & 3 & 4 & 5 & 6 \\ 1 & 2 & 3 & 4 & 5 & 6\end{array}$

Corpo todo:

13. Acatisia

$\begin{array}{llllll}1 & 2 & 3 & 4 & 5 & 6 \\ 1 & 2 & 3 & 4 & 5 & 6 \\ 1 & 2 & 3 & 4 & 5 & 6\end{array}$

1, ausente; 2 , duvidoso; 3 , leve; 4 , moderado; 5 , moderadamente grave; 6 ,grave. 
Tabela 1. Relação entre pacientes internados/discinesia tardia (DT) na cidade de Salvador, Bahia, setembro 1992.

\begin{tabular}{|c|c|c|c|c|c|c|c|}
\hline \multirow{2}{*}{ Hospitais } & \multicolumn{3}{|c|}{ Identificação } & \multicolumn{2}{|c|}{ DT } & \multirow{2}{*}{ Total } & \multirow{2}{*}{$\%$} \\
\hline & $\mathbf{H}$ & $\mathbf{M}$ & Total & $\mathbf{H}$ & $\mathbf{M}$ & & \\
\hline 1. Hospital de Custódia (Manicômio Judiciário) & 263 & 27 & 290 & 6 & 2 & 8 & 2,5 \\
\hline 2. Sanatório Bahia & 198 & 132 & 330 & 4 & 1 & 5 & 1,5 \\
\hline 3. Casa de Saúde Santa Mônica & 334 & 238 & 572 & 3 & 3 & 6 & 1 \\
\hline $\begin{array}{l}\text { 4. Hosp. Universitário Prof. Edgard } \\
\text { Santos/UFBA }\end{array}$ & 5 & 13 & 18 & 0 & 0 & 0 & 0 \\
\hline 5. Sanatório São Paulo & 246 & 114 & 360 & 4 & 4 & 8 & 2 \\
\hline 6. Casa de Saúde Ana Neri & 290 & 87 & 377 & 5 & 0 & 5 & 1,3 \\
\hline 7. Hospital Juliano Moreira & 48 & 120 & 168 & 1 & 2 & 3 & 1,7 \\
\hline Total geral & 1384 & 731 & 2115 & 23 & 12 & 35 & 100 \\
\hline
\end{tabular}

$\mathrm{H}$, homens; M, mulheres.

Tabela 2. Discinesia tardia (DT)/idade.

\begin{tabular}{lcc}
\hline Idade & DT & $\%$ \\
\hline $20-30$ & 0 & 0 \\
$30-40$ & 1 & 2,3 \\
$40-50$ & 6 & 17,3 \\
$50-60$ & 16 & 45,7 \\
$60-70$ & 12 & 34,2 \\
Total & 35 & 100 \\
\hline
\end{tabular}

Todos os pacientes tinham o diagnóstico de esquizofrenia há pelo menos seis meses, com tempo superior a três meses de uso de um dos seguintes neurolépticos: haloperidol, clorpromazina, levopromazina, tioridazina, flufenazina. O paciente mais jovem tinha 20 anos e o mais idoso 68 . O grupo total foi dividido em cinco subgrupos de faixa etária de 10 anos, entre 20 e 70 anos.

\section{RESULTADOS}

Num total de 2115 pacientes, 35 apresentaram critérios clínicos de DT, perfazendo uma prevalênciaponto de $1,65 \%$ (Tabela 1$)$. A maior prevalência $(2,5 \%)$ foi observada no Manicômio Judiciário, enquanto nenhum paciente com DT foi encontrado no Hospital Universitário. O hospital psiquiátrico com maior quantidade de pacientes internados (572 pacientes) apresentou a segunda menor prevalência (1.0\%).

Dos 35 pacientes com DT, 23 eram do sexo masculino e o restante do sexo feminino (Tabela 1). Comparando-se os pacientes com DT e a população não afetada, observamos que a incidência no sexo masculino foi $1,66 \%$, enquanto no sexo feminino corresponde a $1,64 \%$. Do exposto, concluímos não haver significância estatística na incidência entre os sexos.

Quanto os pacientes foram avaliados de acordo com sua faixa etária (Tabela 2), observamos que nenhum paciente com menos de 30 anos apresentara DT. A partir dessa faixa etária, a incidência de DT aumentou progressivamente até os 60 anos, havendo a partir de então, redução na incidência, porém sem significância estatística.

\section{COMENTÁRIOS}

Na observação da nossa casuística de 2115 pacientes, internados durante o mês de setembro1992 nos sete hospitais psiquiátricos de Salvador, Bahia, encontramos a taxa de prevalência de $1,6 \%$, enquanto, na literatura consultada, a taxa média é de $24 \%$. Apesar de ser inferior à taxa média referida, encontra-se no universo de variação de 0,5 a $70,4 \%$, utilizada pela literatura. Esta grande variação da prevalência deve-se à ausência de critérios universalmente aceitos para o diagnóstico da DT $^{6}$. Um problema particular são os casos duvidosos. Em nossa casuística, afastamos este erro, em virtude de termos usado a Escala de Simpson com a pontuação mínima de 3, fato este que explica a baixa prevalência registrada. 
A maior prevalência ocorrida nos sete hospitais em estudo prende-se ao Manicômio Judiciário, onde os pacientes permanecem maior período de tempo internados sob uso prolongado de neurolépticos. $\mathrm{O}$ fato de não termos encontrado pacientes com DT no Hospital Universitário pode ser atribuído ao período de curta internação e ao uso controlado de neurolépticos.

Quanto ao sexo, encontramos 1,6\% no sexo masculino e 3,1\% no feminino. De acordo com o nosso universo de estudo, não existe significância estatística. A literatura estudada aponta um maior número de DT no sexo feminino ${ }^{6,7}$.

Analisando a faixa etária, a DT surgiu a partir dos 30 anos, aumentando progressivamente até os 60 , fato este também relatado por outros autores ${ }^{5,9}$

\section{REFERÊNCIAS}

1. Andrade LAF, Bertolucci PHF, Pereira JS. Discinesia tardia. Arq Neuropsiquiatr 1984, 42: 362-369.

2. Asnis GM, Sachar EJ, Langer G et al. Normal prolactin response in tardive dyskinesia. Psychopharmacology 1979, 66: 247-250.

3. Crane G. Persistent dyskinesia . Br J Psychiatry 1973, 122: 395-405.

4. Faerchtein L. Discinesia tardia; um alerta para o uso indiscriminado dos neurolépticos. J Bras Psiq 1983, 6: 389-398.

5. Feste DV, Wyatt RJ. Changing epidemiology of tardive dyskinesia; an overview. Am J Psychiatry 1981, 138: 3.

6. Kane JM, Smith JM. Tardive dyskinesia; prevalence and risk factors, 1959 to 1979. Arch Gen Psychiatry 1982, 39: 473-481.

7. Mardsen CD. Clinical aspects of the dyskinesias. In: Bradford MF, Mardsen CD (ed) London: Academic Press, 1976, p 3-11.

8. Piedade RAM, Nardi AE. Discinesia tardia: estudo epidemiológico em pacientes esquizofrênicos ambulatoriais. J Bras Psiq 1988, 37: 81-86.

9. Smith MJ, Baldessarini RJ. Changes in prevalence, severity and recovery in tardive dyskinesia with age. Arch Gen Psychiatry 1980, 37: 1368-1373.

10. Tarsy D, Baldessarini RJ. The tardive dyskinesia syndrome. In Klawans HL (ed) Clinical neuropharmacology. New York: Raven Press, 1976, Vol 1, p 29-61.

11. Tetelbom M, Vargas FJ, Osório CMS. Discinesia tardia: um diagnóstico de exclusão. Rev Psiquiatr RS (Porto Alegre) 1991, 2: 92-96. 